\section{0-km-length distributed fiber optical loss sensor based on transmission-reflection analysis}

\author{
Vasilii V. Spirin, MEMBER SPIE \\ Centro de Investigación Científica y de \\ Educación Superior de Ensenada \\ Division de Fisica Aplicada \\ Apartado Postal Número 2732 \\ CP 22860 Ensenada, Baja California \\ Mexico
}

E-mail: vaspir@cicese.mx

Pieter L. Swart, MEMBER SPIE

Anatoli A. Chtcherbakov

Rand Afrikaans University

Electrical Engineering Department

PO Box 524

Auckland Park 2006

Republic of South Africa

\section{S. V. Miridonov \\ Mikhail Shlyagin \\ Centro de Investigación Científica y de \\ Educación Superior de Ensenada \\ Division de Fisica Aplicada \\ Apartado Postal Número 2732 \\ CP 22860 Ensenada, Baja California \\ Mexico}

Abstract. We report, for the first time to our knowledge, the detection and localization of a loss-inducing perturbation along more than 20-km-long test fiber based on the analysis of transmitted and Rayleigh backscattered powers of an unmodulated continuous-wave light source. Localization of a strong disturbance with an estimated accuracy of $1 \mathrm{~m}$ near the source end and $7.5 \mathrm{~m}$ near the remote end of the 21.067-km-long single-mode sensing fiber is demonstrated. (C) 2005 Society of Photo-Optical Instrumentation Engineers. [DOI: $10.1117 / 1.1883585]$

Subject terms: distributed fiber optical sensors; Rayleigh scattering.

Paper L040726 received Oct. 1, 2004; revised manuscript received Dec. 7, 2004; accepted for publication Jan. 21, 2005; appeared online Jan. 31, 2005; published online Apr. 6, 2005.

\section{Introduction}

Distributed fiber optical loss sensors are very attractive for the measurement of pressure, temperature, displacement, etc. ${ }^{1-2}$ Recently, we reported a novel, effective, and potentially inexpensive method for localization of a lossinducing perturbation in a few meter ${ }^{3}$ - and a few kilometer-length ${ }^{4,5}$ optical fiber, based on the measurement of transmitted and reflected, or Rayleigh backscattered, powers of an unmodulated light source. The basic idea of the proposed method is to localize the perturbation by using the unique relationships between normalized transmitted and Rayleigh backscattered powers for different locations of the loss-induced disturbance along the sensing fiber. Indeed, in general, for the identical loss-induced perturbations the backscattered power depends on the location of the excess loss region.

The method can be implemented for the detection of one or several temporally successive, alarm-like perturbations. ${ }^{4}$ Meanwhile, for some applications, it is important to detect and localize alarm conditions, which typically occur as single infrequent events, at very long distances. In this paper, we present a result of localizing loss-inducing perturbations by using transmission-reflection analysis (TRA) in a 21.067-km-length test fiber. This technique can be useful, for example, for lengthy pipe leak detection systems.

\section{Experimental Results and Discussion}

The schematic diagram of the TRA distributed fiber-optic sensor is shown in Fig. 1. Continuous-wave light emitted by a super-luminescent diode operating near a $1550-\mathrm{nm}$ wavelength with a line width of about $50 \mathrm{~nm}$ was launched through an optical circulator into a 21.067-km-length SMF-28 single-mode fiber. The launched optical power was about $0.5 \mathrm{~mW}$, and the attenuation coefficient of the test fiber measured with optical time-domain reflectometry was $0.2 \mathrm{~dB} / \mathrm{km}$. The optical isolator cancelled any back reflections from the remote end of the test fiber. A dual-channel lightwave meter was used to measure the transmitted and Rayleigh backscattered powers. To induce excess losses in the sensing fiber, we used bending transducers, shown schematically in Fig. 1. The losses were induced by bending approximately $2-3 \mathrm{~mm}$ of test fiber at different positions. By tuning the bending transducer, we could change the transmitted power by more than $-20 \mathrm{~dB}$ from its initial undisturbed value.

Figure 2 presents the calculated relationships between normalized reflected power and the square of the normalized transmitted power for the excess loss induced at distances $l_{n}=n \Delta L$ from the source end of the test fiber, where $n=0,1 \ldots 10$, and the interval between bending locations $\Delta L=2106.7 \mathrm{~m}$. Transmitted and reflected powers were normalized with respect to their initial undisturbed values. The main contributor to reflected power (more than 90\%) is Rayleigh backscattering phenomena in the optical fiber. However, weak residual reflections from the source end and the remote end of the sensing fiber, which were equal to $1.3 \times 10^{-5}$ and $2.1 \times 10^{-5}$, respectively, were also taken into account in the calculations. The relationships are linear

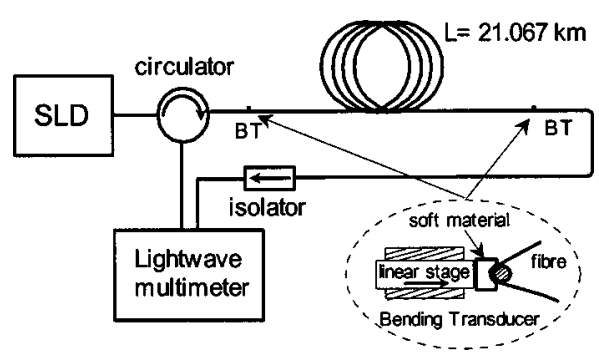

Fig. 1 Schematic diagram of the distributed TRA fiber-optic sensor. 
E

$\mathbf{L}$

E

$\mathrm{T}$

$\mathrm{T}$

E

$\mathbf{R}$

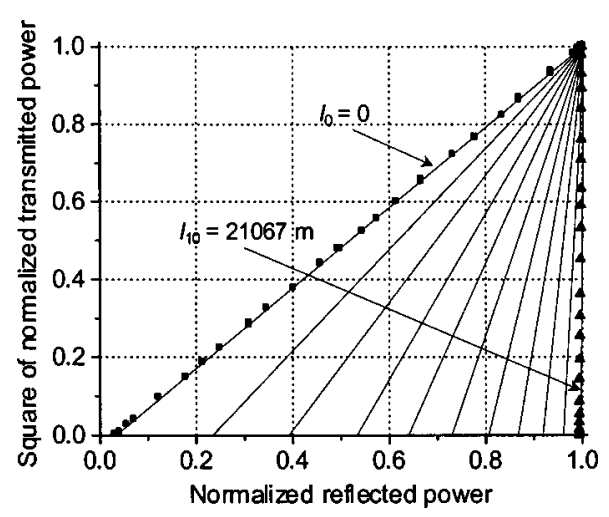

Fig. 2 Relationships between normalized reflected and square of normalized transmitted powers for losses induced at different locations along 21.067-km-length test fiber. Solid lines: calculated data, $\boldsymbol{\square}$ and $\boldsymbol{\Lambda}$ : experimental data.

for a perturbation that affects the test fiber at any location. The unique slope of the relationship depends on the position of the perturbation along the test fiber. Hence, the localization of the perturbation with the TRA method can be performed by evaluating the slope of the dependence of normalized reflected power versus the square of normalized transmitted power. Density of the lines for equally separated locations of the perturbations as seen in Fig. 2 shows the potential accuracy of the localization with the TRA method. With this method it is easier to localize a strong perturbation near the source end of the test fiber compared with a weak perturbation near the remote end of the test fiber. Figure 2 also shows experimental dependencies between normalized reflected and the square of normalized transmitted powers for the additional losses induced near the source and remote ends of the test fiber. The experimental data perfectly coincide with the calculated values. Note, that in the calculations we did not use any fitting parameters.

Figure 3 shows the relationships between normalized reflected and the square of the normalized transmitted power for the excess losses induced at two different points separated by $15 \mathrm{~m}$ near the remote end of the $21.067-\mathrm{km}$-test fiber. As we can see, only $40 \%$ excess losses induced at two different locations near the remote end produced distinct responses, and therefore can be clearly recognized. The localization error due to random variation of power measurements for the strong perturbations near the remote end of the test fiber was estimated as $7.5 \mathrm{~m}$. All experimental measurements were carried out at stable ambient conditions with the temperature variation not exceeding $\pm 1^{\circ} \mathrm{C}$. In order to study the temperature influence, the spool with $21.067-\mathrm{km}$-length test fiber was placed inside a laboratory oven. The ratio between normalized reflected $R_{n}$ and the square of the normalized transmitted $T_{n}^{2}$ powers changes by more than $10 \%$ with the temperature varying from $+15^{\circ} \mathrm{C}$ to $+60^{\circ} \mathrm{C}$. The increase of the ratio was mainly due to a decrease in the transmitted power. The total reflected power was changed by less than $0.4 \%$ over the above mentioned temperature range. For our experimental configuration, the variation of the transmission with temperature depends on

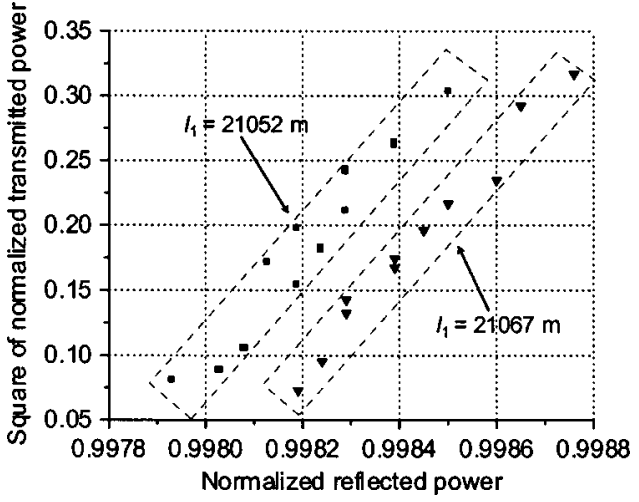

Fig. 3 Relationships between normalized reflected and square of normalized transmitted powers for losses induced near the end of a $21.067-\mathrm{km}$-length fiber, at two locations separated by $15 \mathrm{~m}$.

coil parameters and fiber winding conditions. The noticeable decrease of the transmission in the experiment was likely due to non-optimal winding conditions of the fiber on the spool. In practice, however, test fiber should be placed inside a special cover. The optimal design of the TRA sensing cable can provide practical independence of the transmission and reflection on the temperature inside a moderate working range of the sensor, because both the Rayleigh backscattered and transmitted powers depend very weakly on the temperature. ${ }^{6,7}$

\section{Conclusion}

We have demonstrated that a distributed fiber-optic sensor based on the TRA principle provides localization of disturbances that decreased the initial transmission by at least $40 \%$ with a maximum localization error of $\pm 7.5 \mathrm{~m}$ along 21.067-km-length test fiber.

\section{Acknowledgments}

We gratefully acknowledge financial support from Telkom SA, ATC (Pty) Ltd., Marconi Communications South Africa (Pty) Ltd., and THRIP.

\section{References}

1. A. Hartog, "Distributed fiber-optic sensors: principles and applications," in Optical FiberSensor Technology, Advanced ApplicationsBragg Gratings and Distributed Sensors, K. T. V. Grattan and B. T. Meggitt, Eds., Kluwer Academic Publishers, Boston (2000).

2. D. A. Nolan, P. E. Blaszyk, and E. Udd, "Optical fibers," in Fiber Optic Sensors: An Introduction for Engineers \& Scientists, John Wiley \& Sons, New York (1991).

3. V. V. Spirin, M. G. Shlyagin, S. V. Miridonov, and P. L. Swart, "Transmission/reflection analysis for distributed optical fibre loss sensor interrogation," Electron. Lett. 38, 117-118 (2002).

4. V. V. Spirin, "Transmission-reflection analysis for localization of temporally successive multi-point perturbations in distributed fiber-optic loss sensor based on Rayleigh backscattering," Appl. Opt. 42, 11751181 (2003).

5. V. V. Spirin, F. J. Mendieta, S. V. Miridonov, M. G. Shlyagin, A. A. Chtcherbakov, and P. L. Swart, "Localization of a loss-inducing perturbation with variable accuracy along a test fiber using transmissionreflection analysis," IEEE Photonics Technol. Lett. 16, 569-571 (2004).

6. M. E. Lines, "Scattering losses in optic fiber materials. I. A new parameterization," J. Appl. Phys. 55, 4052-4057 (1984).

7. Y. Li, F. Zhang, and T. Yoshino, "Wide temperature-range Brillouin and Rayleigh optical-time-domain reflectometry in a dispersionshifted fiber," Appl. Opt. 42, 3772-3775 (2003). 